\title{
Stochastic simulation of acoustic communication in turbulent shallow water
}

\section{Bjerrum-Niese, Christian; Lutzen, $\mathbf{R}$.}

\section{Published in:}

I E E E Journal of Oceanic Engineering

Link to article, DOI:

$10.1109 / 48.895360$

Publication date:

2000

Document Version

Publisher's PDF, also known as Version of record

Link back to DTU Orbit

Citation $(A P A)$ :

Bjerrum-Niese, C., \& Lutzen, R. (2000). Stochastic simulation of acoustic communication in turbulent shallow water. I E E E Journal of Oceanic Engineering, 25(4), 523-532. https://doi.org/10.1109/48.895360

\section{General rights}

Copyright and moral rights for the publications made accessible in the public portal are retained by the authors and/or other copyright owners and it is a condition of accessing publications that users recognise and abide by the legal requirements associated with these rights.

- Users may download and print one copy of any publication from the public portal for the purpose of private study or research.

- You may not further distribute the material or use it for any profit-making activity or commercial gain

- You may freely distribute the URL identifying the publication in the public portal

If you believe that this document breaches copyright please contact us providing details, and we will remove access to the work immediately and investigate your claim 


\title{
Stochastic Simulation of Acoustic Communication in Turbulent Shallow Water
}

\author{
Christian Bjerrum-Niese and René Lützen
}

\begin{abstract}
This paper presents a stochastic model of a turbulent shallow-water acoustic channel. The model utilizes a Monte Carlo realization method to predict signal transmission conditions. The main output from the model are statistical descriptions of the signal-to-multipath ratio (SMR) and signal fading. Probability density functions of signal envelope are evaluated by Pearsons's Skew-Kurtosis Chart, generally predicting Ricean fading. Dynamic calculations of SMR by the model overcome the main inconveniences of deterministic calculations, providing "smooth" instead of "noisy" curves as a result. Dynamic calculations of SMR and fading are concluded to provide more intelligible and realistic results than deterministic calculations.
\end{abstract}

Index Terms-Signal-to-multipath ratio, stochastic modeling, turbulence, underwater communication.

\section{INTRODUCTION}

$\mathbf{C}$ OHERENT acoustic communication has been receiving considerable attention in recent years. The use of this technology has obvious applications in shallow water, yet there is a tendency of failure of the communications systems in the horizontal shallow-water channel. On the other hand, the successful implementations often depend highly on the local environment. Hence, such prototype systems are not suitable for commercialization.

Present focus is placed on very shallow water as one of the most challenging communication channels. Examples of this type of channel are the North Sea, the Baltic Sea, and the inner Danish waters connecting these seas. These channels are characterized by seasonally dependent stratification. The sound speed profile is perturbed by mixing caused by currents, freshwater run-off, and weather conditions.

The aim of this paper is to develop a simulation tool to be used for estimation of the performance of acoustic communications systems in a variety of shallow-water channels. The primary output of this model is a parameter to classify the quality of the communication channel. The simulation should provide further insight into the physical limitations of the channel and how the system should be designed to overcome these limitations. Therefore, emphasis is placed on a physically realistic channel model rather than by studying receiver architectures.

This paper is organized as follows. The development of the simulation tool will be related to previous studies in Section II.

Manuscript received January 7, 1998; revised December 14, 1999. This work was supported in part by the Danish Technical Research Council (STVF).

C. Bjerrum-Niese is with the Department of Industrial Acoustics, Technical University of Denmark, 2800 Lyngby, Denmark.

R. Lützen is with Ødegaard \& Danneskiold-Samsøe A/S, 2100 Copenhagen, Denmark.

Publisher Item Identifier S 0364-9059(00)06639-5.
The features of a ray model and the establishment of the performance evaluation parameter are presented in Section III. Next, the theory for introducing channel dynamics through turbulence-induced sound speed fluctuations is presented in Section IV. The turbulence model is related to the propagation model in Section V. The study is completed in Section VI by a numerical case study including statistical tools to describe the pressure field fluctuations. Conclusions are drawn in Section VII.

\section{MODELING OF THE UNDERWATER COMMUNICATION CHANNEL-A REVIEW}

A simulation tool to model the communication link in shallow water may be of value during both the system design phase and operations in the field. A model will always be inaccurate to some degree due to model imperfection and to input parameter uncertainties. Therefore, a model should not be used for optimization but rather to provide guidelines and trends. While designing a system, a simulation tool can be used to test different system configurations. When operating the system in the field, this tool may explain poor performance of the link, for instance, that the receiver depth should be changed-if possible.

When developing a realistic model, it is important to include the most critical features. The limitations of the shallow-water communication channel are described by two features: intersymbol interference (ISI) and signal fading. The multipath propagation of sound will cause a time spread of the impulse response of the channel, causing ISI. The dynamic properties of the channel cause a frequency spread of every spectral component of the transmitted signal, leading to amplitude and phase fluctuations.

Several approaches have recently been made to simulate a communication system. Studies emphasizing a realistic model of the complex propagation conditions will be reviewed in the following. The physical properties of the multipath channel are conveniently related to the signal characteristics of the communication system through the establishment of the time-varying impulse response function of the underwater medium.

The channel impulse response function should be related to the source and receiver functions, as well as to the signal modulator and demodulator, and possibly also to further receiver processing modules. This concept was examined by Bjerrum-Niese et al. in [1]. A detailed model was presented which included all the features mentioned above in a modular structure. The output of this model is a synthetic time - series generator to be used as an alternative to in situ recordings. The drawback of this strategy is, however, the difficulties in pointing out the limiting factor 
and relating it to the acoustical properties of the channel, because no reduction of the data output was attempted.

A similar, though somewhat simpler, model was presented by Essebar and Vercelloni [2]. Temporal variations due to relative motion between the source and receiver are included in this model. However, the physical aspects of the communication channel are only modeled for a single environmental case. Essebar et al. [3] model the shallow-water communication channel by use of the ray method. The seasonal variations of time spread and transmission loss are examined and found to be considerable. They claim frequency spread to be negligible and hence ignore all dynamic features of the channel. In our opinion, this assumption is questionable.

Galvin and Coates include fluctuations in their shallow-water ray model [4]. These temporal fluctuations are modeled as being induced by rough surface variability, specifically by claiming the amplitude of the scattered sound to be Rayleigh-distributed and the phase to be Gaussian-distributed. The assumption of Rayleigh-distributed amplitude may hold for specific cases, but not in general. This approach was extended in [5], where a procedure to generate random time series of prescribed spectral density and probability density functions is derived specifically for a Rayleigh channel. A random series generator such as this is useful for testing receiver processing architectures. On the other hand, the study of the physical processes to generate the fluctuations is not emphasized.

Plaisant [6] and Cristol [7] studied the sound field when transmitting with a parametric array in deep water over a long range. They included internal waves as a dynamic feature and directly solved the transport equation in the ray method. Though this work may prove to be important, it is presently limited to the inclusion of the upper part of the internal wave spectrum in deep water, and, therefore, it does not apply to the present study of shallow-water propagation.

Assuming that the channel is not noise-limited but rather multipath-limited, a convenient parameter to describe the quality of the communication channel is the signal-to-multipath ratio (SMR). Zielinski et al. [8], [9] introduce the SMR parameter to quantify the ISI of the communication channel. Their propagation model is a deterministic sound speed ray model. Impressive performance of a communication system is predicted, which is surely due to the simplified propagation model.

The approach of evaluating the multipath channel through the SMR parameter was further developed by Bjerrum-Niese and Bjørnø in [10]. Here, a deterministic refractive ray model rather than a straight line (constant sound speed) ray model was used to model the impulse response function. Predictions of optimum receiver depths were sought. The numerical results were, however, difficult to analyze due to strong fluctuations of the SMR. This can be explained by the fact that a deterministic ray model only provides a snapshot image of the channel with an interference pattern of many rays leading to arbitrary transmission loss at the receiver.

Stochastic features induced by a time-varying surface reflection coefficient was examined by Bjerrum-Niese in [11]. The prediction of a stochastic SMR became possible through a realization technique using a randomly varying surface. However, this technique is only useful if a dominating part of the transmitted sound has interacted with the sea surface. For a strongly downward refracting channel, the dynamic sea surface will have little influence.

This fact has motivated the present study, where the medium rather than the sea surface is modeled as time-varying. Internal waves have little influence in the shallow seas of interest in this study. The channel is characterized by the significant temporal variability of both the temperature and salinity and the corresponding stratification. This has led to the development of a stochastic propagation model based on turbulence-induced sound speed fluctuations. Other dynamic features such as rough surface variability and relative motion between the source and receiver are purposely not included here, not because they are unimportant, but rather to be able to study the physical features independently. Extensions to include these features may be implemented in a straightforward manner following [1], for instance.

\section{Propagation Model And Performance Evaluation}

This section will present the deterministic propagation model. Next, we carry out the evaluation of the communication system performance by computation of the SMR. To compute the SMR and the dynamic features presented in Section $\mathrm{V}$, the ray method given here is not the only approach to model the sound propagation.

\section{A. The Deterministic Ray Model}

A numerical ray model has been implemented. To improve efficiency of the model, the propagation algorithm is semianalytical. It applies solutions for sound speed profiles that are piecewise linear in depth and range-independent, i.e., the paths follow either straight lines or circular arcs.

Boundary interaction is very important in the shallow-water channel. It is not unlikely that even the strongest paths are refracted and have been multiply reflected or scattered from either the bottom or the sea surface. Surface loss is modeled as deterministic, knowing that it is actually stochastic. This was chosen to separate the dynamic influence of the physical features. Hence, we have chosen to model surface loss by introducing a wind-driven absorptive bubble layer, following an empirical expression obtained by Dahl [12].

Bottom interaction is modeled by assuming that the bottom will be a reflector with a complex impedance given by the sound speed, density, and absorption coefficient. Changes in the bathymetry and small-scale roughness are ignored.

The output of the deterministic ray model will be the impulse response function of the channel $h(t)$. It is assumed to be a sum of delta functions with complex amplitude $a_{i}$ and delay $\tau_{i}$ :

$$
h(t)=\sum_{i=1}^{N} a_{i}(t) \delta\left(t-\tau_{i}\right) .
$$

By identifying all eigenrays for a specific channel geometry, the ray model output is an estimate of all coefficients $\left(a_{i}, \tau_{i}\right)$. 


\section{B. Performance Evaluation Through the Signal-to-Multipath Ratio}

This section will present the tools for analyzing the output of the ray model in terms of parameters that may evaluate the performance of a communication system. The SMR introduced by Zielinski et al. [9] is a convenient measure for evaluating the communication link quality of the channel. This algorithm was further analyzed in [10] and [11] and is presented here in a slightly modified version.

Corresponding to the impulse response function of the channel, every delayed version of the transmitted symbol will corrupt the received signal. This is known as ISI. The corruption is proportional to the ratio of the delay to the symbol period $T_{s}$. Two parameters are formed: the signal strength $S$ and the multipath strength $M$.

The signal strength $S$ is defined as the amplitude of the coherent sum of the direct path and the self-multipath, where the self-multipath is the part of the multipath structure that is present within the time frame of the currently received symbol

$$
S=\left|\sum_{i=1}^{r}\left(1-\frac{\tau_{i}}{T_{s}}\right) s_{i}\right|
$$

where $r$ is the number of self-multipaths, each represented as a phasor, $s_{i}=a_{i} e^{2 \pi i f \tau_{i}}$, and $\tau_{i}$ is taken w.r.t. the strongest arrival.

The corruptive multipath strength $M$ is defined as the coherent sum of delayed multipaths, i.e., the tail of the previously received signal present in the time frame of the current signal denoted the cross-multipath. The definition of multipath strength is the amplitude of the coherent sum of interference from the adjacent symbol and of previous signals with delays $\tau_{i}>T_{s}$ :

$$
M=\left|\sum_{i=2}^{r} \frac{\tau_{i}}{T_{s}} s_{i}+\sum_{i=r+1}^{N} s_{i}\right| .
$$

Error-free transmission can be shown [21] to be obtained when the SMR is sufficiently large as

$$
\mathrm{SMR}_{\mathrm{QPSK}}=\frac{S}{M}>\sqrt{2}
$$

for quasi-phase shift keying (QPSK) modulation (four phase levels). The general requirement for error-free transmission for $(n) \mathrm{PSK}$ is

$$
\operatorname{SMR}_{(n) \operatorname{PSK}}>\frac{1}{\cos \left(\pi / 2-\frac{\pi}{n}\right)} .
$$

\section{ChANNEl DyNAMiCS}

The stochastic model is an extension to the deterministic ray model. The geometrical definition of the acoustic channel is the same for both models.

The shallow-water channel is divided into $p$ layers (Fig. 1). Each layer corresponds to a certain turbulence structure characterized by its parameter of local relative sound speed fluctuation $C_{\mu}$. For each layer, a maximum turbulence length scale $L_{0}$ is assumed to be equal to the height of the layer.

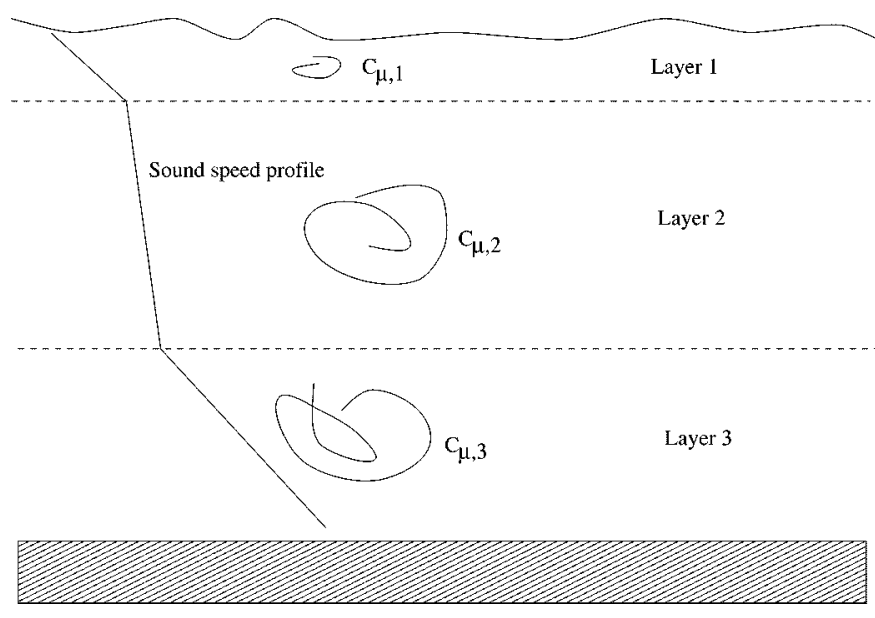

Fig. 1. A three-layer turbulent acoustic channel model.

\section{A. Converting Turbulence to Sound Speed Fluctuations}

Assuming range independence, the sound speed can be expressed as

$$
\begin{aligned}
& c(t, z)=c_{0}(z)(1+\mu(z, t)) \\
& \mu(z, t)=\frac{\Delta c}{c_{0}}=\frac{1}{n(z, t)}-1
\end{aligned}
$$

where $t$ is the time, $z$ the depth, and $c_{0}$ the mean sound speed profile. Also, $\mu(z, t)$ is a measure of the local relative sound speed fluctuation, which is related to the refractive index $n$. The distribution of $\mu$ is assumed Gaussian, being a fair assumption according to [13].

According to [13]-[15], the three-dimensional (3-D) spectrum $F_{\mu}$ of the local relative sound speed fluctuations in a homogeneous and locally isotropic turbulent layer can be represented by

$$
\begin{aligned}
F_{\mu}(k) & =0.033 \cdot C_{\mu} k^{-11 / 3} \\
\frac{2 \pi}{L_{0}} & \ll k \ll \frac{2 \pi}{l_{0}}
\end{aligned}
$$

where $k$ is a wavenumber $k=2 \pi / \lambda$ representing the turbulence length scale. The structure parameter $C_{\mu}$ represents the level of fluctuation and has dimensions $\left[m^{-2 / 3}\right] . L_{0}$ and $l_{0}$ are the outer and inner scales of the turbulence, referred to as the energycontaining scale and the dissipation scale, respectively. In [14], (8) was found to well describe a turbulent channel.

Also, the turbulence may be split into two parts, one regarding fluctuations due to the flow velocity and the other regarding temperature and salinity fluctuations. Thus, the structure parameter is [14] (slightly modified after consulting the authors)

$$
C_{\mu}=\frac{11}{6} C_{\mu, \text { velocity }}+C_{\mu, \text { temp.+salin. }}
$$

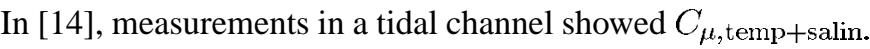
of the order $O \sim 0.25 \cdot C_{\mu \text {,velocity }}$ at most, and generally less. The order of magnitude, however, depends on the oceanographical conditions. In this text, to include effects from velocity, temperature, and salinity fluctuations, $C_{\mu}$ is defined as

$$
C_{\mu} \approx\left(\frac{11}{6}+a\right) C_{\mu, \text { velocity }}
$$


where $a$ is an order of magnitude parameter in the range 1 . $10^{-1} \leq a \leq 1 \cdot 10^{1}$. The local relative sound speed fluctuation spectrum $F_{\mu}$ and the turbulent energy dissipation rate $\epsilon(z)$ are related by [14] assuming homogeneous and locally isotropic turbulence and by using (9)

$$
\begin{aligned}
F_{\mu, \text { velocity }}(k, z) & =\frac{1.5 \cdot \epsilon(z)^{2 / 3} k^{-5 / 3}}{4 \pi c_{0}(z)^{2} k^{2}} \\
& =0.033 \cdot\left(\frac{11}{6} C_{\mu, \text { velocity }}(z)\right) k^{-11 / 3} \\
\Rightarrow \quad F_{\mu}(k, z) & \approx 0.033 \cdot C_{\mu}(z) k^{-11 / 3}
\end{aligned}
$$

where

$$
C_{\mu}(z)=\left(\frac{11}{6}+a\right) \cdot 11.364 \cdot \frac{\epsilon(z)^{2 / 3}}{\pi c_{0}(z)^{2}} .
$$

$F_{\mu}$ has the dimensions of $\left[\mathrm{m}^{3}\right]$. In the above expression, $c_{0}(z)$ is the value of the mean sound speed profile at the depth considered. The turbulent energy dissipation rate $\epsilon$ and the sound speed profile are the fundamental input parameters of this modeling approach.

A mean value of $\epsilon$ and $c_{0}$ will be assigned to a particular layer. If the dissipation rate $\epsilon(z)$ and the upper limit of the turbulence length scale $L_{0}$ are known together with the mean sound speed profile, $\mu$ is described statistically by integrating the 3-D spectrum of $\mu$. The integration limits are the wavenumbers given by the upper length scale $L_{0}$ and the lower length scale $l_{0}$, respectively. However, in actual turbulence, $l_{0}$ is very small and the corresponding energy is negligible [14]. Hence, the wavenumber representing $l_{0}$ is assumed to be infinity.

This integration is performed in [14], and, together with (11), it leads to

$$
\begin{aligned}
\left\langle\mu^{2}\right\rangle & =4 \pi \cdot 0.033 \cdot C_{\mu} \int_{k_{0}=2 \pi / L_{0}}^{\infty} k^{-5 / 3} d k \\
& =\left(\frac{11}{6}+a\right) \frac{2.25 \cdot \epsilon^{2 / 3}}{c_{0}^{2}}\left(\frac{L_{0}}{2 \pi}\right)^{2 / 3} .
\end{aligned}
$$

\section{B. Distribution of Dissipation Rate $\epsilon$}

The physical meaning of the dissipation rate $\epsilon$ (dissipation rate per unit mass $\left.\left[\mathrm{m}^{2} / \mathrm{s}^{3}\right]\right)$ is the rate by which turbulent kinetic energy is dissipated, i.e., converted to heat.

In the case of a negligible rate of change in the control volume and transportation of turbulent kinetic energy across the boundaries (this is the case of turbulent boundary layers [14]), the entire production of turbulent energy equals the dissipation rate. In this modeling approach, the key assumption is that the entire turbulent energy production is dissipated. Furthermore, effects from medium inhomogeneities such as wave-induced bubble plumes are ignored.

Referring to Fig. 2, the following observations are made.

- At the surface, the wind will supply energy to the water mass; $\epsilon(z)$ thus assumes a high value at the surface. Due to the viscosity of the water, the energy produced at the surface will be transmitted to the molecules beneath, but with dissipation losses. An expression for $\epsilon(z)$ is given in

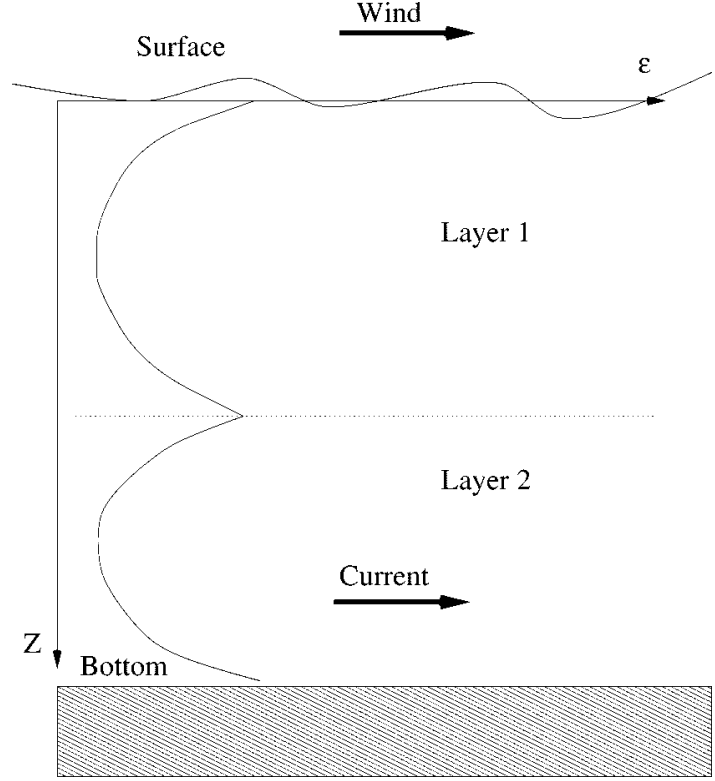

Fig. 2. Exemplified variation of the dissipation rate $\epsilon$ in a two-layer stratified channel.

[16] in the surface boundary layer measured in the North Sea.

- At the stratification interface, two fluids of different densities meet. Due to the friction between the layers and the constant motion of the molecules, the fluids will mix at the interface. This mixing of fluids causes turbulence to arise and $\epsilon(z)$ will assume a high value at the interface, decreasing with the distance from the interface due to the viscosity. No analytical expression of $\epsilon(z)$ for this particular case has been found in the literature, but $\epsilon(z)$ will be assumed to decrease proportionally to $d^{-1}, d$ being the distance from the interface. This assumption is based on the similarity of the viscous effects in the interface boundary layer and in the surface and bottom boundary layers (following [17]).

- At the bottom of the channel, a turbulent boundary layer will grow as at the other interfaces. Turbulent energy will arise from the friction between the fluid and the bottom. In [16], an expression for $\epsilon(z)$ in the bottom layer is given, measured in the North Sea.

\section{Converting Sound Speed Fluctuations to TRAVEL-TIME FLUCTUATIONS}

This section deals with the stochastic time delay $\Delta t_{i}$ caused by one layer alone. A more detailed presentation is given in [18]. A ray traveling through a nonfluctuating medium spends an amount of time $t_{0, i}$ in a layer $i$. This deterministic travel time is calculated by the deterministic ray model. In the case of a fluctuating medium, the travel time through the $i$ th layer becomes

$$
t_{i}=t_{0, i}+\Delta t_{i}
$$

Thus, when the medium causes $\Delta t_{i}$ to fluctuate, so will $t_{i}$. This leads to a probability density distribution of $t_{i}$ with mean $t_{0, i}$. 

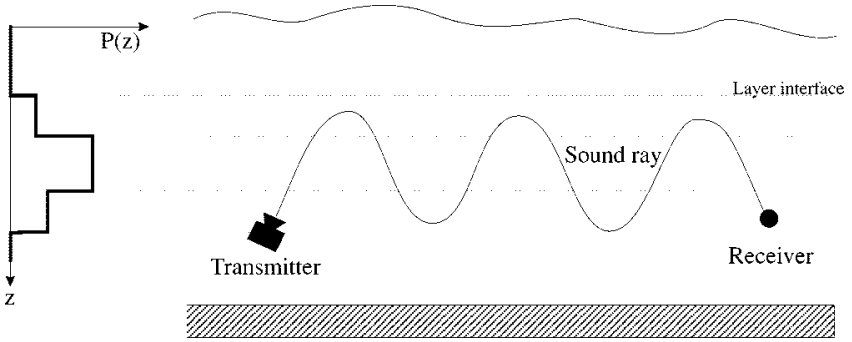

Fig. 3. Outline of a ray and the corresponding probability function $P(z)$.

\section{A. Probability Function}

The above mentioned $t_{i}$ contains information on the duration of the passage of the ray through layer $i$. This duration corresponds to a fraction of the total distance. As a useful tool, we introduce the probability function $P(z)$ illustrated in Fig. 3 that contains information on the fraction of the passage through a particular layer, measured in meters, relative to the total traveled distance $L_{\text {tot }}$. Hence, $P(z)$ is derived only from the geometry of the ray. Thus, $P_{i}=12 \%$ means that the ray spends $12 \%$ of its journey in layer $i$. It should be noted that $L_{\mathrm{tot}}$ is only used to make $P$ nondimensional and may therefore be chosen at one's convenience. In this case, $L_{\text {tot }}$ is chosen so that $\sum_{i=1}^{p} P_{i}=1$.

For a ray traveling in a straight line from $\left(x_{1}, y_{1}\right)$ to $\left(x_{2}, y_{2}\right)$, the definition of $P$ is

$$
P=\frac{L}{L_{\mathrm{tot}}}=\frac{\sqrt{\left(x_{2}-x_{1}\right)^{2}+\left(y_{2}-y_{1}\right)^{2}}}{L_{\mathrm{tot}}} .
$$

For a ray traveling in a circular arc of radius $R_{0}$ from $\left(x_{1}, y_{1}\right)$ to $\left(x_{2}, y_{2}\right)$ (see Fig. 4 ), the definition of $P$ is

$$
\begin{aligned}
& P=\frac{S}{L_{\mathrm{tot}}}= \frac{R_{0} \cdot\left|\theta_{2}-\theta_{1}\right|}{L_{\mathrm{tot}}}, \\
& \theta=\left\{\begin{array}{cc}
\pi-\arcsin \frac{y}{R_{0}}, & x>0 \wedge y>0 \\
\pi+\arcsin \frac{y}{R_{0}}, & x>0 \wedge y<0 \\
\arcsin \frac{y}{R_{0}}, & x<0
\end{array}\right\} .
\end{aligned}
$$

\section{B. $\Phi-\Lambda$ Space}

Following [13] and assuming isotropic turbulence as in [14], the fluctuations of the acoustic medium are described by the two parameters $\Phi$ and $\Lambda$. These characterize fluctuation strength and the size of the inhomogeneities, respectively. With $q_{0}=(2 \pi / \lambda)$ the acoustic wavenumber, $L_{c}$ the correlation length (defined in Section V-C), and $L_{\text {tot }}$ the total distance traveled by the ray, the definition of the parameters becomes

$$
\begin{aligned}
\Phi^{2} & \approx q_{0}^{2}\left\langle\mu^{2}\right\rangle L_{\text {tot }} \cdot 0.4 \cdot L_{c} \\
\Lambda & \approx \frac{L_{\text {tot }}}{6 \cdot L_{c}^{2} q_{0}} .
\end{aligned}
$$

The size parameter $\Lambda$ measures the influence of diffraction of the acoustic wave.

A $\Phi-\Lambda$ diagram is shown in Fig. 9. In the saturated region $\left(\Lambda \cdot \Phi^{2}>1\right)$, each transmitted ray is split into several rays,

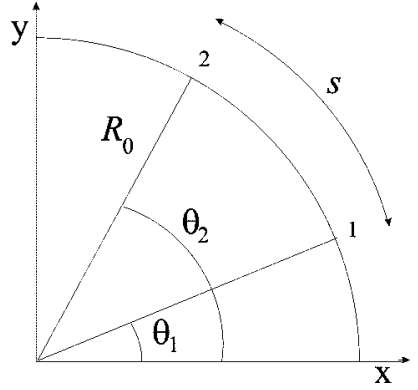

Fig. 4. Illustration of the arclength $S$.

so-called micromultipaths (the number of micromultipaths per ray is approximately $\Phi \cdot \Lambda$ ). The ray approximation will not hold due to the existence of several micromultipaths.

In the unsaturated region $\left(\Lambda \cdot \Phi^{2}<1\right)$, the transmitted ray remains undivided. In the geometrical acoustics regime $(\Lambda \ll 1$, $\Lambda \cdot \Phi^{2} \ll 1$ ) perturbations cause vertical displacements of only small fractions of the correlation length. In the diffractive but unsaturated region $(\Lambda>1, \Phi<1)$, the ray approximation breaks down due to diffraction.

Generally, the interface between saturated and unsaturated regions forms an upper performance bound for coherent acoustic communication [19].

The validity area of the fluctuation model coincides with that of the ray model. Therefore, $\Phi$ and $\Lambda$ must be calculated for each simulation to relate it to the validity area in $\Phi-\Lambda$ space, which is the area of the geometrical acoustics approximation.

\section{C. $\Phi-\Lambda$ Method}

According to [13], the structure coefficient of homogeneous and isotropic turbulence is defined by

$$
C_{\mu}=2 \cdot\left\langle\mu^{2}\right\rangle L_{c}^{-2 / 3} .
$$

Using $\left\langle\mu^{2}\right\rangle$ from (12) and $C_{\mu}$ from (11), the correlation length of the turbulence is calculated from the expression

$$
L_{c}^{2 / 3}=\frac{2 \cdot\left\langle\mu^{2}\right\rangle}{C_{\mu}} .
$$

This correlation length is an important parameter in the definition of the strength parameter $\Phi$ and the size parameter $\Lambda$.

Using the probability function defined in Section V-A, $\Phi$ and $\Lambda$ are related to a particular layer $i$ by

$$
\begin{aligned}
\Phi_{i}^{2} & \approx q_{0}^{2}\left\langle\mu_{i}^{2}\right\rangle P_{i} \cdot L_{\mathrm{tot}} \cdot 0.4 \cdot L_{c, i} \\
\Lambda_{i} & \approx \frac{P_{i} \cdot L_{\mathrm{tot}}}{6 \cdot L_{c, i}^{2} q_{0}} .
\end{aligned}
$$

In the case of the geometrical acoustics approximation, the applicability of $\Phi$ and $\Lambda$ is due to the relation [13]:

$$
\Phi_{i}^{2} \approx\left\langle\phi_{i}^{2}\right\rangle
$$

where $\phi_{i}$ is the phase in radians of the received signal. In the case $\Lambda_{i} \ll 1, \phi_{i}$ has a Gaussian probability distribution [13]. Hence, the probability distribution of $t_{i}$ is Gaussian as well. With $f$ as 
the frequency of the signal, this determines the distribution of $\Delta t_{i}$

$$
\left\langle\left(\Delta t_{i}\right)^{2}\right\rangle=\left(\frac{1}{2 \pi f}\right)^{2}\left\langle\phi_{i}^{2}\right\rangle .
$$

Joining the set of equations (17), (19), and (20), the $\Phi-\Lambda$ method becomes

$$
\left\langle\left(\Delta t_{i}\right)^{2}\right\rangle=\left(\frac{1}{2 \pi f}\right)^{2}\left(q_{0}^{2}\left\langle\mu_{i}^{2}\right\rangle P_{i} L_{\mathrm{tot}} \cdot 0.4 L_{c, i}\right)
$$

in which, using (11), (12), and (16),

$$
\begin{aligned}
L_{c, i}^{2 / 3} & =\frac{2 \cdot\left\langle\mu_{i}^{2}\right\rangle}{C_{\mu, i}} \\
\Rightarrow L_{c, i} & =0.2208 \cdot L_{0, i}
\end{aligned}
$$

and

$$
\left\langle\mu_{i}^{2}\right\rangle=\left(\frac{11}{6}+a\right) \frac{2.25 \cdot \epsilon_{i}^{2 / 3}}{c_{0, i}^{2}}\left(\frac{L_{0, i}}{2 \pi}\right)^{2 / 3} .
$$

As for amplitude fluctuations of a transmitted ray, the work in [13] states that, within the geometrical acoustics region, the pressure amplitude $A$ is log-normal distributed. Thus, amplitude fluctuations are calculated from

$$
\begin{aligned}
u & =\ln A \\
\left\langle u^{2}\right\rangle & \approx 1 / 4 \cdot \Lambda \Phi^{2} .
\end{aligned}
$$

Therefore, the log-amplitude $u$ is a Gaussian-distributed random variable. As mentioned above, this method is confined to the case $\Lambda \ll 1, \Lambda \cdot \Phi^{2} \ll 1$, i.e., high frequency, limited transmission range, and small sound speed fluctuations.

\section{Combination of Layered Travel-Time Fluctuations}

Each layer of the medium contributes to the resulting stochastic part of the time of arrival (t.o.a) $\Delta t_{\text {t.o.a. }}$ by a Gaussiandistributed $\Delta t$. The layered channel is modeled as a series of stationary time-varying filters acting subsequently on the transmitted signal. Thus, the superposition of subsequent layers/filters is achieved by convolving their impulse responses.

The medium is assumed linear so that the time delays from each layer may be added. Since the filters are linear, the filter calculations will be reduced. The filtering of one layer is only performed once, although the ray may have passed at different times.

The $\left\langle\Delta t_{i}^{2}\right\rangle$ 's of the different layers are zero-mean Gaussiandistributed random variables. Thus, the superpositions will also be Gaussian and zero-mean, and only the second-order moment $\left\langle\Delta t_{i}^{2}\right\rangle$ of the resulting distribution is left to be expressed.

It follows from the linearity that a simple addition of the $\left\langle\left(\Delta t_{i}\right)^{2}\right\rangle$ 's of the different layers is sufficient to describe the resulting distribution of the $\Delta t_{\text {t.o.a. }}$. Thus,

$$
\left\langle(\Delta t)_{\text {t.o.a. }}^{2}\right\rangle=\left\langle(\Delta t)_{1}^{2}\right\rangle+\left\langle(\Delta t)_{2}^{2}\right\rangle+\cdots+\left\langle(\Delta t)_{p}^{2}\right\rangle .
$$

For the $\Phi-\Lambda$ method, the second-order moment of the stochastic part of the t.o.a. $\left\langle(\Delta t)_{\text {t.o.a. }}^{2}\right\rangle$ becomes

$$
\left\langle(\Delta t)_{\text {t.o.a. }}^{2}\right\rangle=\sum_{i=1}^{p}\left(\frac{1}{2 \pi f}\right)^{2}\left(q_{0}^{2}\left\langle\mu_{i}^{2}\right\rangle P_{i} \cdot L_{\text {tot }} \cdot 0.4 \cdot L_{c, i}\right)
$$

in which $L_{c, i}$ is given by (22) and $\left\langle\mu_{i}^{2}\right\rangle$ by (12).

The method is valid for $\Lambda_{\mathrm{tr}} \ll 1$ and $\Lambda_{\mathrm{tr}} \cdot \Phi_{\mathrm{tr}}^{2} \ll 1$ (tr $\sim$ transmission). Here, $\Lambda_{\operatorname{tr}}$ is the largest of all $\Lambda_{i}$ while $\Phi_{\operatorname{tr}}$ is the largest of all $\Phi_{\text {ray }}=\sum_{1}^{p} \Phi_{i}$, where $p$ is the number of layers.

\section{NUMERICAL RESULTS}

\section{A. Implementation of the Fluctuation Model}

The above described $\Phi-\Lambda$ method is implemented as a fluctuation model using Monte Carlo simulation. The procedure is as follows.

1) Deterministic impulse response and propagation data are calculated by a deterministic ray model.

2) From turbulence and propagation data, oceanographical parameters $\Phi$ and $\Lambda$ are assigned to each transmitted ray.

3) Phase and log-amplitude variances for each ray are determined from $\Phi$ and $\Lambda$.

4) The deterministic impulse response is repeatedly perturbed slightly according to Monte Carlo realizations of amplitude and phase. For each realization, thte signal envelope and SMR are evaluated.

5) Finally, the set of realizations (typically $O \sim 10^{4}$ ) is subjected to statistical analysis.

\section{B. Ricean Statistics and Pearsons's Skew-Kurtosis Chart}

We introduce some statistical concepts that are used in relation to the fluctuation model. The probability density function of the Ricean distribution, of which both the Gaussian and Rayleigh distributions can be treated as special cases, is reviewed in the Appendix.

The use of Pearsons's Skew-Kurtosis Chart is a technique to classify the unknown PDF of a fluctuating signal. It is particularly useful for modeling purposes where stationarity and convergence are controlled. It is based on the calculation of the skew and kurtosis, defined as

$$
\begin{aligned}
\text { Skew: } & \beta_{1}=\frac{E_{c}\left\{X^{3}\right\}^{2}}{E_{c}\left\{X^{2}\right\}^{3}} \\
\text { Kurtosis: } & \beta_{2}=\frac{E_{c}\left\{X^{4}\right\}}{E_{c}\left\{X^{2}\right\}^{2}}
\end{aligned}
$$

where $X$ is the stochastic variable and $E_{c}\left\{X^{p}\right\}$ is the central moment of order $p$.

In this work, skew and kurtosis will be calculated from amplitude PDF's and plotted on the Pearsons's Chart, thus facilitating comparison with the above mentioned theoretical distributions. An example is shown in Fig. 13.

\section{Numerical Test Case}

To demonstrate the features of the fluctuations model, the channel configuration shown in Table I was simulated. 
TABLE I

CHANNEL PARAMETERS

\begin{tabular}{l|c}
\hline \hline Channel depth & $60 \mathrm{~m}$ \\
\hline Source depth & $50 \mathrm{~m}$ \\
\hline Range & $4000 \mathrm{~m}$ \\
\hline Bottom material & Muddy sand \\
\hline Wind speed & $6 \mathrm{~m} / \mathrm{s}$ \\
\hline Signal carrier frequency & $30 \mathrm{kHz}$ \\
\hline Symbol rate & $3 \mathrm{ksymb} / \mathrm{s}$ \\
\hline Parameter of order of magn. $a$ & 0.25 \\
\hline Max. turbulence length scale, $0-10 \mathrm{~m}$ & $10 \mathrm{~m}$ \\
\hline Max. turbulence length scale, $10-15 \mathrm{~m}$ & $5 \mathrm{~m}$ \\
\hline Max. turbulence length scale, $15-60 \mathrm{~m}$ & $45 \mathrm{~m}$ \\
\hline Number of horizontal layers in model & 4 \\
\hline \hline
\end{tabular}

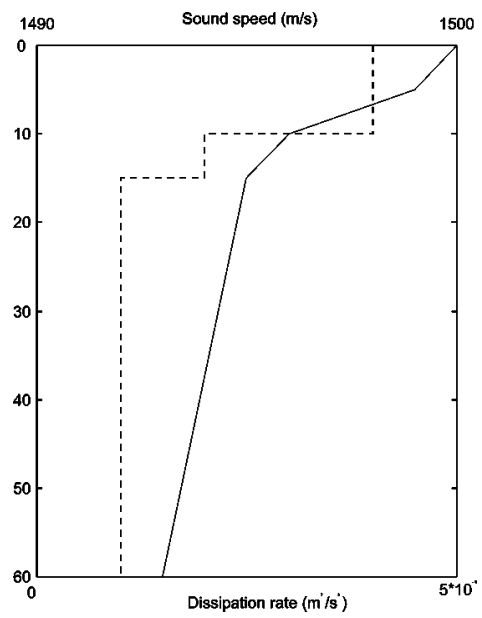

Fig. 5. Sound speed profile and dissipation rate profile, leading to four layers in the model. Solid line: sound speed; dashed line: dissipation rate.

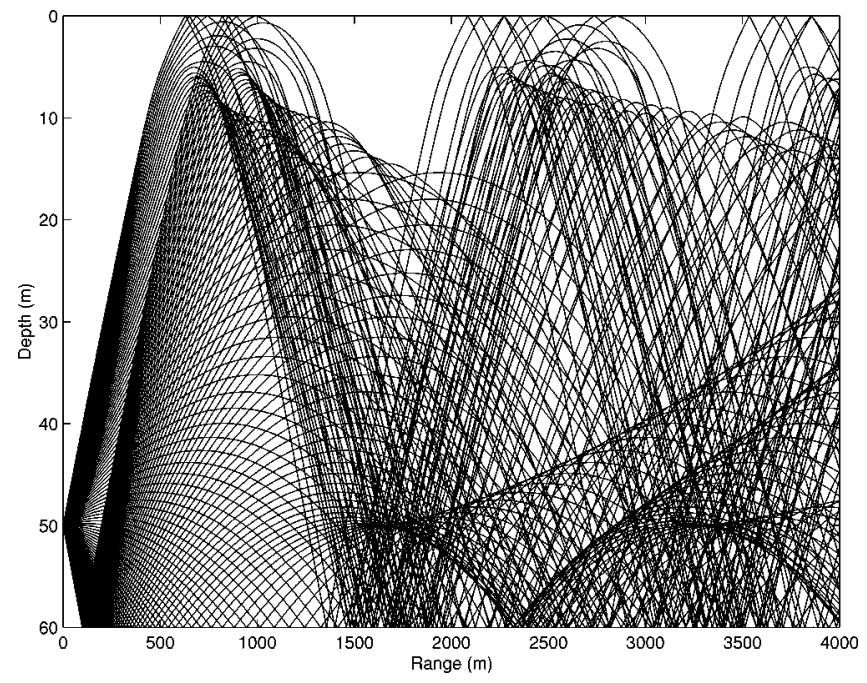

Fig. 6. Deterministic raytrace.

The corresponding sound speed profile and turbulent dissipation rate profile are shown in Fig. 5. Both represent approximations to typical profiles found in the North Sea (see Section IV-B). It is apparent from the deterministic raytrace in Fig. 6 that the channel provides a severe multipath environment for communication purposes. The transmission loss, shown in Fig. 7, is about 70-80 dB, expect within the near-surface bubble

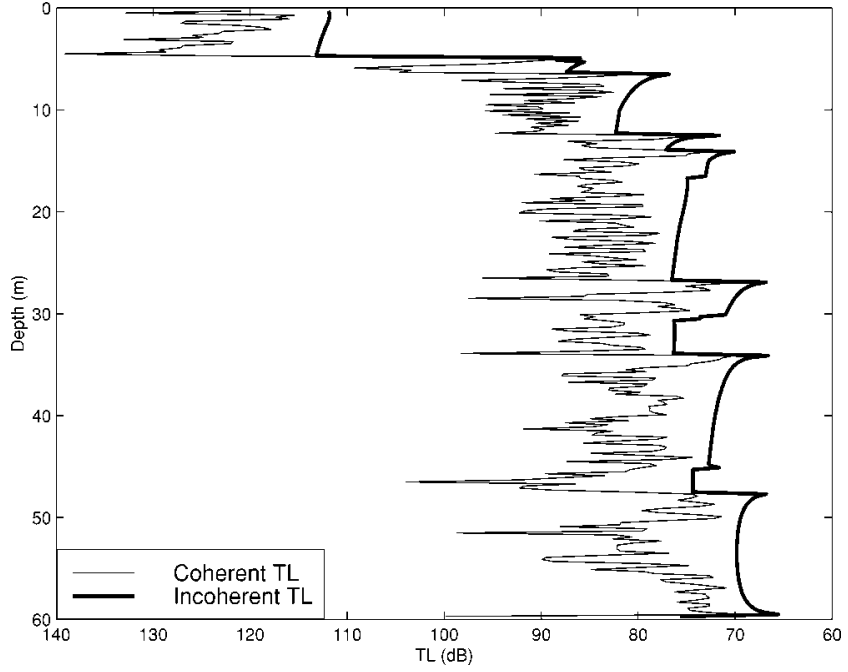

Fig. 7. Transmission loss curve (rays summed coherently and incoherently). The spikey nature of the incoherent transmission loss arises from the caustics observed in Fig. 6.

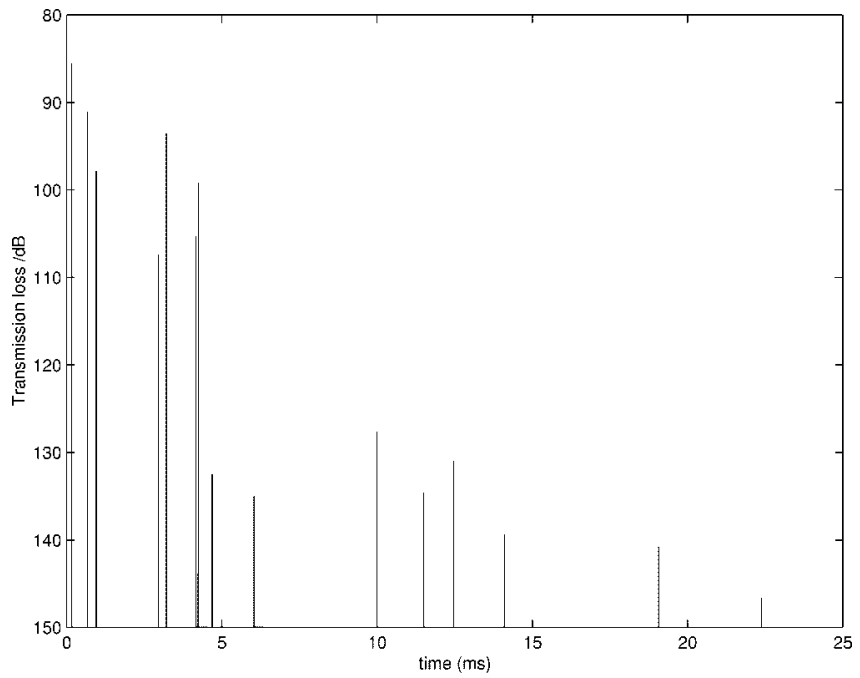

Fig. 8. Deterministic channel impulse response for a receiver at $50 \mathrm{~m}$.

layer. This fact indicates that a standard commercial acoustic transmitter (source level $>180 \mathrm{~dB}$ re $1 \mu \mathrm{Pa}$ ) will provide a sufficient signal-to-noise ratio (SNR).

1) Impulse Response, SMR Realizations, and PDF's for One Depth of the Receiver: Simulations of transmission with the receiver positioned $50 \mathrm{~m}$ below the surface are described in the following. The model simulates transmission of a QPSK modulated signal having a pressure amplitude of 1 (dimensionless). The deterministic transmission loss curve is shown in Fig. 7 and the impulse response function is shown in Fig. 8. The transmission at this particular depth of receiver is characterized by a few strong arrivals followed by several weaker arrivals. Hence, Ricean fading is expected according to [20]. Oceanographical parameters $\Phi$ and $\Lambda$ for the transmission (marked by an " $X$ ") are shown in Fig. 9. It is seen that the transmission is inside the validity area of the model, which is the geometrical acoustics region. Each "o" in the figure represents the passage of one ray through a layer of the channel. Each group of o's represents a 


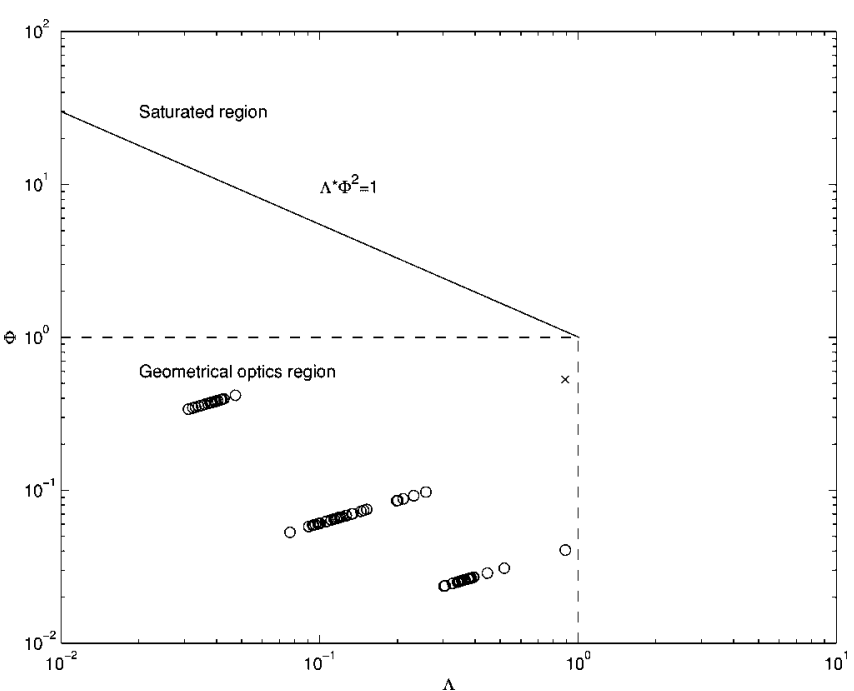

Fig. 9. $\Phi-\Lambda$ diagram for a receiver at $50 \mathrm{~m}$. Three groups of o's are observed corresponding to each turbulent layer.

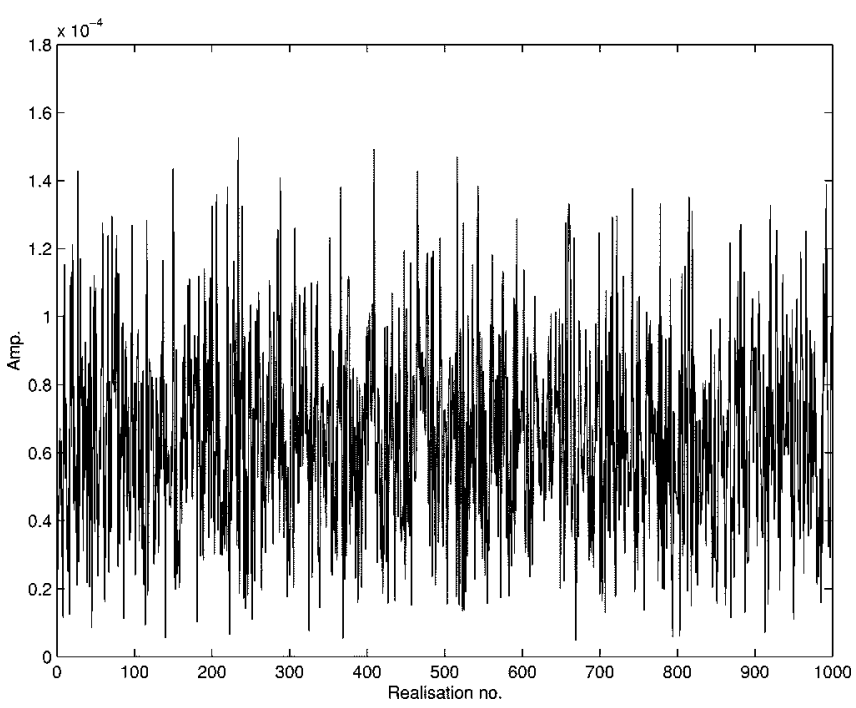

Fig. 10. Realizations of the envelope for a receiver at $50 \mathrm{~m}$.

layer, in which the $\Phi$ 's and $\Lambda$ 's of the rays only differ a little in $L_{\text {tot }}$ and $P$ due to different ray angles.

Now, realizations of signal transmission are performed according to the $\Phi-\Lambda$ method described above. A subset of the resulting envelope realizations is shown in Fig. 10 and statistical analysis of the entire set gives the Ricean PDF in Fig. 11 with randomicity $T=0.44$.

2) Stochastic versus Deterministic SMR Calculations: When SMR is calculated through the deterministic propagation for all receiver depths, the curve becomes rather "noisy" (see Fig. 12). The numerous peaks are caused by local constructive or destructive interference of the rays.

Before performing stochastic calculations of the mean SMR versus depth, oceanographical parameters $\Phi$ and $\Lambda$ were calculated for all depths and were found to be sufficiently limited as required in Section V-B. At this point, 10000 realizations of the SMR were performed for each depth of the receiver in steps of $0.2 \mathrm{~m}$ down through the channel. The result is plotted in Fig. 12. The following observations are made. The stochastic

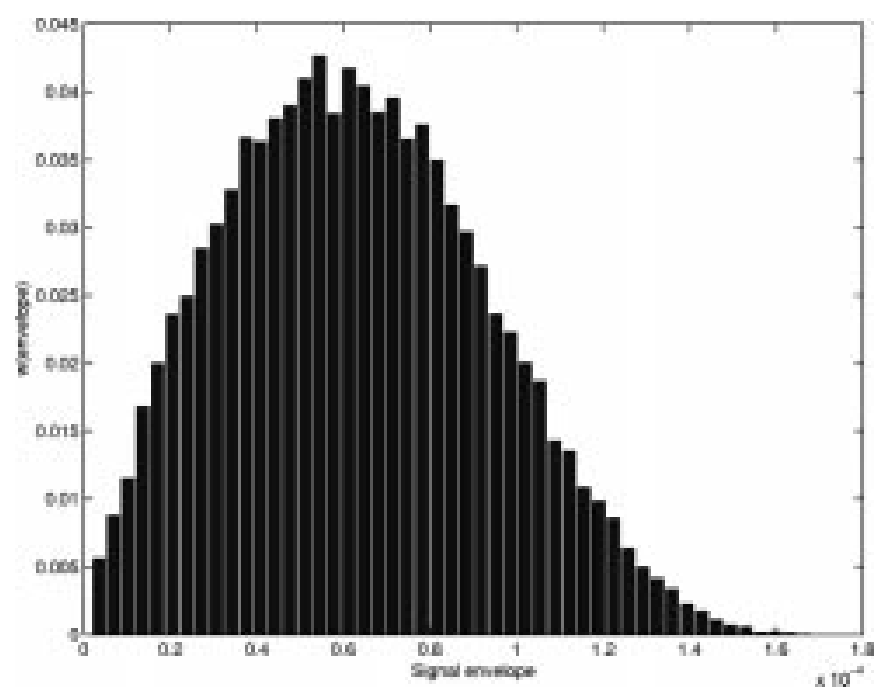

Fig. 11. Envelope PDF for a receiver at $50 \mathrm{~m}$, over 10000 realizations.

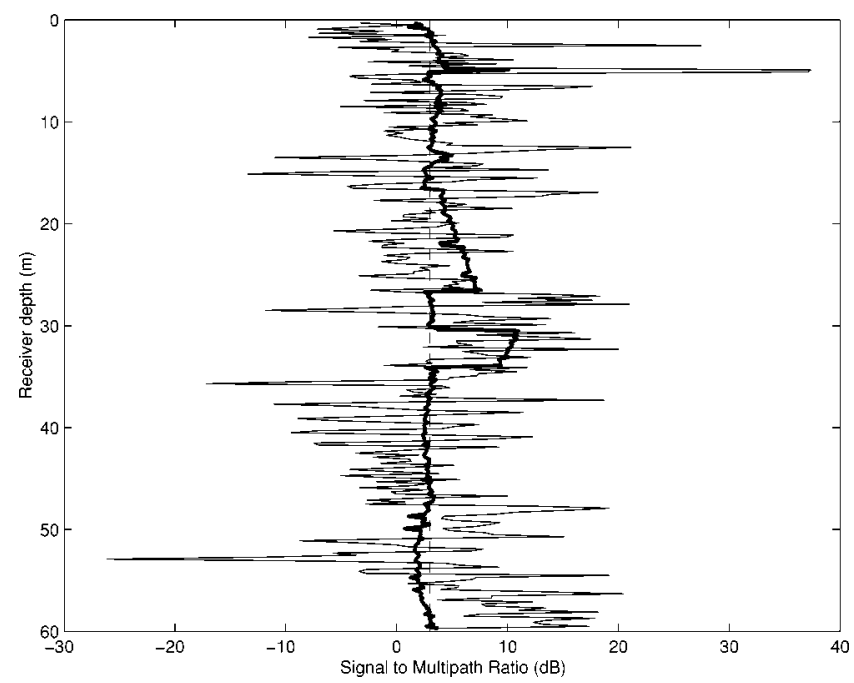

Fig. 12. Stochastic mean and deterministic SMR for all receiver depths, 10000 realizations, and a data rate of $6 \mathrm{kbit} / \mathrm{s}$. Solid bold line: stochastic SMR; solid line: deterministic SMR; dashed line: QPSK threshold $(\sqrt{2}=3 \mathrm{~dB})$.

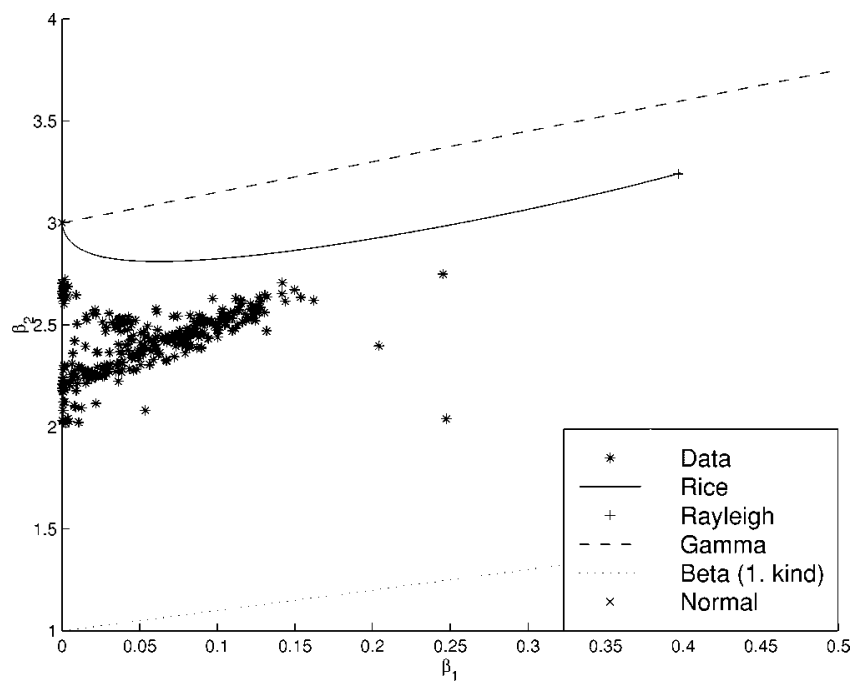

Fig. 13. Pearson's Skew-Kurtosis chart for all receiver depths, 10000 realizations. A few outliers are observed. 


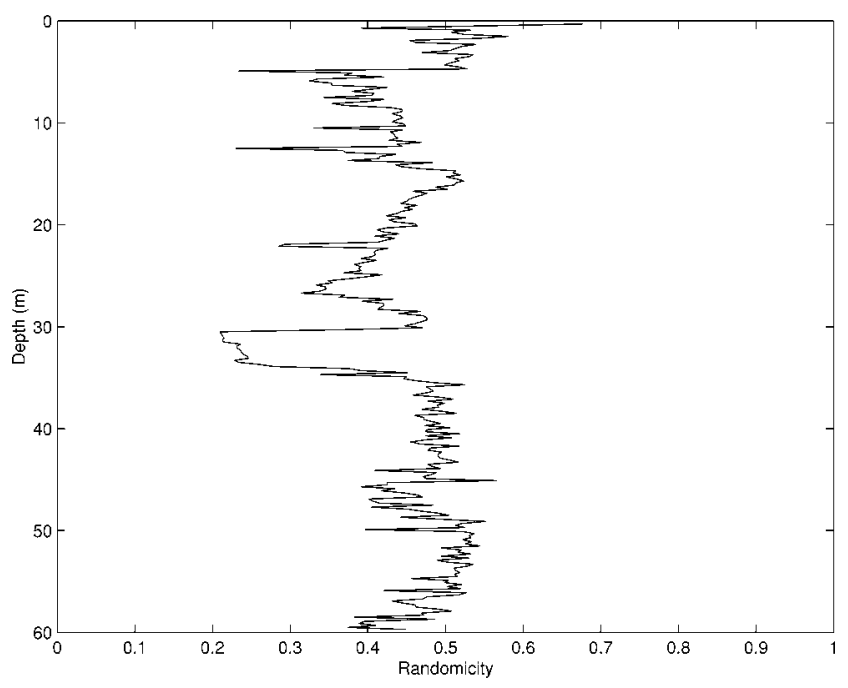

Fig. 14. Randomicity as a function of depth, 10000 realizations.

results $\left(\mathrm{SMR}_{\mathrm{sto}}\right)$ are a "smoothened" version of the deterministic results $\left(\mathrm{SMR}_{\mathrm{det}}\right)$. $\mathrm{SMR}$ sto follows $\mathrm{SMR}_{\mathrm{det}}$, increasing and decreasing at the same intervals of depth. Finally, the level of $\mathrm{SMR}_{\text {sto }}$ is above $\sqrt{2}(3 \mathrm{~dB})$, so that optimum conditions for communication are expected for the receiver at depth intervals between 30-35 m. Skew and Kurtosis were calculated for envelope PDF's for each depth of the receiver and plotted in a Pearsons diagram (see Fig. 13). The envelope simulations appear to follow the theoretical Rice distribution, with Kurtosis somewhat lower than the theoretical one.

Following the simulations, the randomicity $T$ was calculated and is shown in Fig. 14. The numerical case exhibits Ricean fading with randomicity generally below 0.55 . This relatively high degree of randomicity should also be expected from the raytrace in Fig. 6 which indicates no presence of a direct path. The relation between the randomicity distribution and the multiple input parameters is, however, difficult to establish due to the complexity of the problem.

\section{CONCLUSIONS}

A model was presented for the simulation of sound propagation in a turbulent shallow-water channel. This model can be used to determine several critical parameters in relation to acoustic communications under water. The model predicted Ricean amplitude fading with a depth-varying degree of randomicity, consistent with theoretical and experimental expectations. Also, deterministic calculations of SMR were compared to stochastic calculations including fluctuation effects due to turbulence. Where deterministic calculations returned "noisy" curves with many sudden peaks, the corresponding stochastic calculations provided "smoother" curves yielding a simpler interpretation. It is concluded that stochastic simulations of turbulent shallow-water channels provide different, and more realistic, results than deterministic simulations.

Verifying the model is a quite difficult task due to the combination of input parameter sensitivity and model complexity.
The presented model may be extended so as to investigate the influence of other time-varying effects, such as sea surface interaction and moving transducers.

\section{APPENDIX \\ PROBABILITY DENSITY FunCTIONS}

According to [20] and [21], the Ricean PDF is suitable in underwater acoustics for describing signal fading. The Rice PDF includes both the Gaussian PDF and the Rayleigh PDF as extreme cases. It provides a suitable description of statistical samples with both a deterministic part and a stochastic part. The power fraction of the deterministic and random parts are $\mu^{2}$ and $2 \sigma^{2}$, respectively. The analytical expression for the PDF of the stochastic variable $\xi$ is

$$
w(\xi)=\frac{\xi}{\sigma^{2}} e^{-\left(\xi^{2}+\mu^{2}\right) /\left(2 \sigma^{2}\right)} I_{0}\left(\frac{\xi \mu}{\sigma^{2}}\right)
$$

where $I_{0}$ is the zeroth-order Bessel function of the first kind. The Rice PDF is characterized by the randomicity parameter

$$
T=\frac{2 \sigma^{2}}{\mu^{2}+2 \sigma^{2}}
$$

It is easily seen that, for $T \rightarrow 1$, the Rice PDF becomes Rayleigh and that, for low randomicity $T \rightarrow 0$, it becomes Gaussian.

\section{ACKNOWLEDGMENT}

The authors would like to acknowledge the suggestions by $\mathrm{D}$. Dilorio and Profs. L. Bjørnø, F. B. Pedersen, and J. A. Jensen.

\section{REFERENCES}

[1] C. Bjerrum-Niese, L. Bjørnoø, M. Pinto, and B. Quellec, "A simulation tool for high data-rate acoustic communication in a shallow-water, timevarying channel," IEEE J. Oceanic Eng., vol. 211, pp. 143-149, Apr. 1996.

[2] A. Essebar, G. Loubet, and F. Vial, "Underwater acoustic channel simulations for communication," in Proc. Oceans/Osates '94, vol. 3, Brest, France, 1994, pp. 495-500.

[3] A. Essebar and E. Vercelloni, "Simulation of communication system for underwater acoustics," in Proc. Oceans '95, vol. 2, San Diego, CA, 1995, pp. 1204-1207.

[4] R. Galvin and R. Coates, "Analysis of the performance of an underwater acoustic communications system and comparison with a stochastic model," in Proc. Oceans/Osates '94, vol. 3, Brest, France, 1994, pp. $478-482$.

[5] R. Galvin and R. Coates, "A stochastic underwater acoustic channel model," in Proc. Oceans '96, vol. 1, Fort Lauderdale, FL, 1996, pp. 203-210.

[6] A. Plaisant, "Long range acoustic communications," in Proc. Third Eur. Conf. Underwater Acoustics, J. Papadakis, Ed., Heraklion, Greece, 1996, pp. 760-764.

[7] X. Cristol, "Coherence function of transient signals in a randomly fluctuating medium over the background of a highly refracting mean waveguide," in Proc. Third Eur. Conf. Underwater Acoustics, J. Papadakis, Ed., Heraklion, Greece, 1996, pp. 495-500.

[8] A. Zielinski, R. Coates, L. Wang, and A. Saleh, "High rate shallow water acoustic communication," in Oceans '93 Proc., vol. 3, Victoria, BC, Canada, 1993, pp. 432-437.

[9] A. Zielinski, Y. Yoon, and L. Wu, "Performance analysis of digital acoustic communication in a shallow water channel," IEEE J. Oceanic Eng., vol. 20, pp. 293-299, Oct. 1995. 
[10] C. Bjerrum-Niese and L. Bjønø, "Simulated design of an acoustic modem for an AUV in a shallow water channel," in Conf. Proc. Undersea Defence Technology 1996. Swanley, U.K., July 1996, pp. $20-24$.

[11] C. Bjerrum-Niese, "Simulated performance of an acoustic modem in a multipath channel," in Proc. Int. Symp. Hydroacoustics and Ultrasonics, EEA Symposium, A. Stepnowski and E. Kozaczka, Eds, 1997, pp. 271-278.

[12] P. H. Dahl, "Bubble attenuation effects in high-frequency surface forward scattering measurements from FLIP," Applied Physics Laboratory, Univ. of Washington, Tech. Rep. APL-UW TR 9307, 1993.

[13] S. M. Flatté, W. H. Munk, K. M. Watson, and F. Zachariasen, Sound Transmission Through a Fluctuating Ocean. New York, NY: Cambridge Univ. Press, 1979.

[14] D. DiIorio and D. M. Farmer, "Path-averaged turbulent dissipation measurements using high-frequency acoustical scintillation analysis," J. Acoust. Soc. Amer., vol. 96, no. 2, pp. 1056-1069, Aug. 1994.

[15] S. F. Clifford and D. M. Farmer, "Ocean flow measurements using acoustic scintillation," J. Acoust. Soc. Amer, vol. 74, no. 6, pp. 1826-1832, Dec. 1983.

[16] C. Veth, "Turbulence measurements in the stratified central north sea with a laser-doppler velocimeter system," in North Sea Dynamics, J. Sündermann and W. Lenz, Eds. Berlin, Germany: Springer-Verlag, 1983, pp. 412-428.
[17] F. Bo Pedersen, "Dept. Hydrodynamics and Hydraulic Engineering, Technical Univ. of Denmark," unpublished.

[18] R. Lützen, "Acoustic communication with an unmanned submarine," M.Sc. thesis, Dept. of Industrial Acoustics, Technical Univ. of Denmark, 1998.

[19] J. A. Catipovic, "Performance limitations in underwater acoustic telemetry," IEEE J. Oceanic Eng., vol. 15, pp. 205-216, July 1990.

[20] D. Jourdain, "Acoustical propagation analysis in shallow water," in Proc. Oceans/Osates '94, vol. 2, Brest, France, 1994, pp. 278-283.

[21] C. Bjerrum-Niese, "Influence of the dynamic sea surface on underwater acoustic communication," Ph.D. dissertation, Dept. Industrial Acoustics, Technical Univ. of Denmark, 1998.

Christian Bjerrum-Niese, photograph and biography not available at the time of publication.

René Lützen, photograph and biography not available at the time of publication. 